\title{
Sedentarismo y Sobrepeso/Obesidad asociados a la prevalencia de infección por Virus de Papiloma Humano de alto riesgo en mujeres de la zona norte de Bucaramanga
}

\author{
Sedentary lifestyle and overweight/obesity associated with \\ prevalence of high risk human papillomavirus in vulnerable \\ population from north region of Bucaramanga
}

\author{
Raitza Bohorquez-Perez'; Bladimiro Rincón-Orozco²; Ruth Martínez-Vega ${ }^{1}$
}

Forma de citar: Bohorquez-Pérez R, Rincón-Orozco B, Martínez-Vega R. Sedentarismo y Sobrepeso/Obesidad asociados a la prevalencia de infección por Virus de Papiloma Humano de alto riesgo en mujeres de la zona norte de Bucaramanga. Rev Univ Ind Santander Salud. 2019; 51(1): 59-68. doi: http://dx.doi.org/10.18273/revsal.v51n1-2019007 @) (1)

\section{Resumen}

Introducción: Para el desarrollo de cáncer de cuello de uterino la infección con el Virus de Papiloma Humano (VPH) es una causa necesaria. Sin embargo, la eliminación viral está asociada a múltiples factores, algunos modificables y otros no, que varían según las características poblacionales y áreas geográficas. Objetivo: Determinar factores asociados a la prevalencia de infección por virus de Papiloma Humano de alto riesgo en mujeres entre 35-65 años con riesgo moderado o alto de cáncer de cuello uterino de la zona norte de Bucaramanga. Metodología: Estudio analítico de corte transversal. A las mujeres clasificadas como de moderado/alto riesgo para cáncer de cuello uterino en una encuesta corta estandarizada, se les realizó una encuesta sobre factores potenciales asociados a infección, seguida de una autónoma de muestra cérvical, que se utilizó para detección de DNA viral. Se realizó análisis múltiple de regresión log-binomial para obtener Razones de Prevalencia ajustadas (RPa). Resultados: Se entrevistaron 810 mujeres, de éstas 435 tuvieron moderado/alto riesgo para cáncer y se realizaron la autotoma. La prevalencia de infección por VPH de alto riesgo fue de 5,1\% (IC 95\% 3,2-7,6). El sedentarismo (RPa 2,51; IC95\% 1,15-5,50) y el sobrepeso/obesidad (PRa 4,20; IC95\% 1,00-15,51) se asociaron a mayor prevalencia. Además, hubo una tendencia de incremento en la prevalencia de infección en las usuarias de anticonceptivos inyectables; así como una tendencia de disminución de esta prevalencia según porciones de frutas y verduras consumidas diariamente. Conclusión: Factores modificables como sedentarismo y sobrepeso/obesidad, se asociaron independientemente a mayor prevalencia de infección por VPH en la población estudiada.

Palabras clave: Factor de riesgo; Papillomavirus Humano; Alto riesgo; Prevalencia; Sedentarismo; Sobrepeso; Obesidad.

1. Universidad de Santander. Bucaramanga, Colombia.

2. Universidad Industrial de Santander. Bucaramanga, Colombia.

Correspondencia: Bladimiro Rincón Orozco. Dirección: Escuela de Microbiología, Universidad Industrial de Santander Cra. 32 29-31 Bucaramanga. Teléfono: +577 6344000. Correo electrónico: blrincon@uis.edu.co. 


\begin{abstract}
Introduction: Infection with Human Papillomavirus is mandatory for cervical cancer development. However, viral elimination is associated to multiple factors, according to the population characteristics and geographic areas, some are modifiable and others not. Objective: To determine the factors associated with the prevalence of high risk human papillomavirus infection in women between 35-65 years old with moderate or high risk of cervical cancer located at the northern zone of Bucaramanga Methodology: Cross-sectional analytical study. Women classified as moderate/ high risk for cervical cancer in a short standardized survey, were surveyed on the side effects associated to chronic viral infection and self-sampling cervical test. Bivariate analysis and multiple analysis of log-binomial regression were performed. Results: 810 women were interviewed; 435 cases of high cancer risk were presented. The prevalence of high risk human papilloma virus infection was 5.1\% (95\% CI 3.2-7.6). The sedentary lifestyle (RPa $2.51,95 \%$ CI 1.15-5.50) and overweight/obesity (PRa 4.20, 95\% CI 1.00-15.51) were associated with higher prevalence. In addition, there was a trend of increased prevalence of infection among users of injectable contraceptives; as well as a tendency to decrease this prevalence according to portions of fruits and vegetables consumed daily. Conclusion: modifiable factors such as sedentary lifestyle and overweight / obesity were independently associated with a higher prevalence of infection by human high risk papilloma virus in the population studied.
\end{abstract}

Keywords: Risk factors; Papillomavirus Human; Prevalence; Sedentary lifestyle; Overweight; Obesity.

\section{Introducción}

El Virus del Papilloma Humano (VPH) infecta queratinocitos de tejidos epiteliales. Estos virus han coevolucionado junto a su hospedero natural siendo estrictamente específicos en cada una de las especies que infectan, de ellos se han aislados más de 120 genotipos $^{1}$. VPHs que infectan el tracto genital han sido clasificados por la Agencia Internacional para la Investigación y el Cáncer (IARC, por sus siglas en inglés) en dos grupos, bajo riesgo (VPH-BR) y alto riesgo (VPH-AR). Los VPH-AR son 16, 18, 31, 33, 35, 39, 45, 51, 52, 56, 58, 59, 68, y 73. Los VPH-AR están relacionados con cánceres anogenitales, entre ellos el cáncer de cuello uterino $(\mathrm{CCU})$ y una gran proporcion de cánceres de orofaringe ${ }^{2}$.

Se estimó que para el año 2008 CCU fue el tercer cáncer más común en la población femenina en todo el mundo con 530.000 casos nuevos, después de cáncer de mama y de pulmón, y la cuarta causa más común de muerte femenina por cáncer (275.000 muertes) $)^{3}$. El 86\% de los casos incidentes (453.531) y el $88 \%$ de las muertes ocurren en regiones menos desarolladas ${ }^{4}$. Es as $i$ como la mitad de los casos de CCU diagnosticados ocurren en mujeres menores de 50 años de edad y son VPH- 16 y 18 los responsables del $80 \%$ de los casos a nivel mundial ${ }^{5}$.

CCU es multifactorial, sin embargo, para que este ocurra se requiere una infección permanente por VPH$\mathrm{AR}^{6}$. Se han documentado factores que pueden modular la infección viral, por ejemplo, el inicio temprano de las relaciones sexuales ${ }^{6}$, múltiples compañeros sexuales ${ }^{7}$, enfermedades de trasmisión sexual ${ }^{8}$ y uso de anticonceptivos ${ }^{9,10}$, entre otros.

De estos múltiples factores de riesgo descritos en la literatura, algunos de ellos se han asociado a la prevalencia de infección por VPH consistentemente en diferentes poblaciones. Sin embargo, otros factores han mostrado evidencia controversial. Por eso el objetivo de este estudio es determinar factores asociados a la prevalencia de infección por VPH-AR en mujeres de 35 a 65 años con riesgo moderado o alto de CCU de la zona Norte de Bucaramanga.

\section{Materiales y métodos}

Se realizó un estudio analítico de corte transversal donde se incluyeron mujeres entre 35-65 años residentes de la zona norte de Bucaramanga que habían tenido o tenían vida sexual; mujeres en estado de embarazo e histerectomizadas fueron excluidas.

Entre septiembre de 2016 y febrero de 2018 se recolectó la muestra por conveniencia a través de tres estrategias: 1) Jornadas de trabajo de campo programadas con cada líder comunitario de los sectores de la zona I y II del norte de Bucaramanga; 2) Salidas de campo casa a casa, acompañados de líderes que ayudaban a identificar mujeres potencialmente elegibles según los criterios ya establecidos; 3) Se acudió a los seis centros de salud de la zona norte donde se invitó a participar a todas las mujeres que asistían por motivos diversos, y si cumplían los criterios de inclusión, previa voluntariedad, fueron incluidas. 
Seguidamente se aplicó una encuesta corta estandarizada creada por Sisteman Cancer Center que las clasificó como de riesgo moderado y alto para desarrollar CCU. Ésta constaba de los siguientes factores de riesgo: fumar más de 25 cigarrillos al día ( +1 punto), tener relaciones antes de los 16 años ( +2 puntos), haber tenido 3 o más parejas sexuales masculinas $(+3$ puntos), haber tenido uno o más hijos ( +1 punto) o 3 o más hijos ( +2 puntos) $\mathrm{y}$ haber tenido una enfermedad de transmisión sexual como VPH, gonorrea, verrugas o SIDA (+2 puntos). También considera dos factores protectores, como el uso frecuente de preservativo al momento de tener relaciones sexuales ( -2 puntos) y la realización de la citología en los últimos 3 años (-2 puntos).

Luego de aplicar a las mujeres la encuesta corta si alcanzaban un puntaje igual o mayor a tres se continuaba con una encuesta complementaria que buscó identificar los factores asociados a la prevalencia de infección como tabaquismo, antecedentes ginecológicos, consumo de alcohol, actividad física, consumo de frutas y verduras, entre otros. Posterior a esto, se brindaron instrucciones verbales y visuales para que las mismas mujeres se tomaran la muestra con un dispositivo de autotoma de muestra cervico-uterina (cepillo), que se almacenó en seco y a temperatura ambiente para su transporte al Laboratorio Central de Investigación en la UIS donde se realizó la prueba molecular para genotipificación de VPH. Los cepillos de autotoma han mostrado una alta especificidad $(95,9 \%)$, sensibilidad $(92,6 \%)$ y concordancia $(91,2-$ $96,8)$ respecto a la muestra tomada por personal de salud $^{11-14}$.

Se evaluó la variable dependiente infección por VPHAR definida como presencia de ADN viral de VPH-AR por la técnica VPH direct flow Chip kit, con el equipo semiautomático hybriSpot 12TM (HS12). Esta prueba tuvo alta comparabilidad con las pruebas Linear Array y CLART con un porcentaje de acuerdo de $91,5 \%$ y un índice de Kappa de 0,798 y 0,818, respectivamente; y detectó más infecciones totales y múltiples que las pruebas mencionadas ${ }^{15}$. Adicionalmente, mostró un acuerdo del $100 \%$ en un estudio realizado por el Centro de referencia de VPH de la $\mathrm{OMS}^{16}$.

Como variables independientes se incluyeron los potenciales factores descritos previamente, entre estos, el estado civil medido como pareja estable (mujeres casadas y en unión libre) y pareja no definida (solteras, separadas, viudas y divorciadas). La ocupación se consideró dicotómica como mujeres cuya actividad actual se realiza primordialmente en la vivienda (amas de casa, desempleadas y pensionadas) y las que se dedican a alguna actividad laboral fuera de casa (empleadas e independientes). Dentro de los síntomas vaginales presentes en el momento de la encuesta se incluyeron flujo, flujo de mal olor, rasquiña, sangrado, entre otros.

El sedentarismo se generó a partir de la variable actividad física total utilizando la fórmula de minutos en la semana invertidos en actividades moderadas, más dos veces los minutos por semana invertidos en actividades vigorosas. De esta manera sedentarismo se definió cuando la mujer realizó menos de $150 \mathrm{~min}$. de actividad física total por semana según la recomendación de la $\mathrm{OMS}^{17}$. Además, se midieron la talla y el peso, el IMC calculado se categorizó según la clasificación de la OMS en sobrepeso con un IMC $\geq 25$ pero $<30$, obesidad con IMC $\geq 30$, y normal para aquellas mujeres con IMC $>18,5$ pero $<25$. En este grupo se ubicó una mujer con un IMC menor de 18,5, es decir de bajo peso.

Para el análisis de las variables cualitativas se emplearon frecuencias absolutas y frecuencias relativas, para las variables cuantitativas se utilizó la mediana y rango intercuartil porque las variables no presentaron distribución normal. Posteriormente, se calcularon las razones de prevalencias (RP) con el intervalo de confianza del $95 \%$ (IC95\%) y el valor $p$. Las variables que tuvieron un valor $p<0,20$ fueron evaluadas en un modelo de regresión múltiple log-binomial para obtener las razones de prevalencias ajustadas $(\mathrm{RPa})$. El análisis se realizó con el Software Stata 12.

\section{Resultados}

Se realizaron 810 encuestas de tamización a mujeres que cumplieron con los criterios de inclusión y que no presentaron criterios de exclusión. De estas, 435 mujeres tuvieron un puntaje moderado o alto para CCU, por lo cual se les realizó detección de VPH-AR a través de autotoma de muestra del cuello uterino. De estas mujeres, 16 presentaron infección por VPH-BR, ocho mujeres presentaron genotipos indeterminados, una muestra no pudo ser procesada por presencia de abundante sangre y negación por parte de la mujer para repetir la muestra, 22 presentaron infección por VPHAR y en 388 no se detectó ADN viral (Figura 1.). Los genotipos de VPH-AR detectados fueron VPH-39, 31, $52,58,59,73,56,68,16$ y 45. 


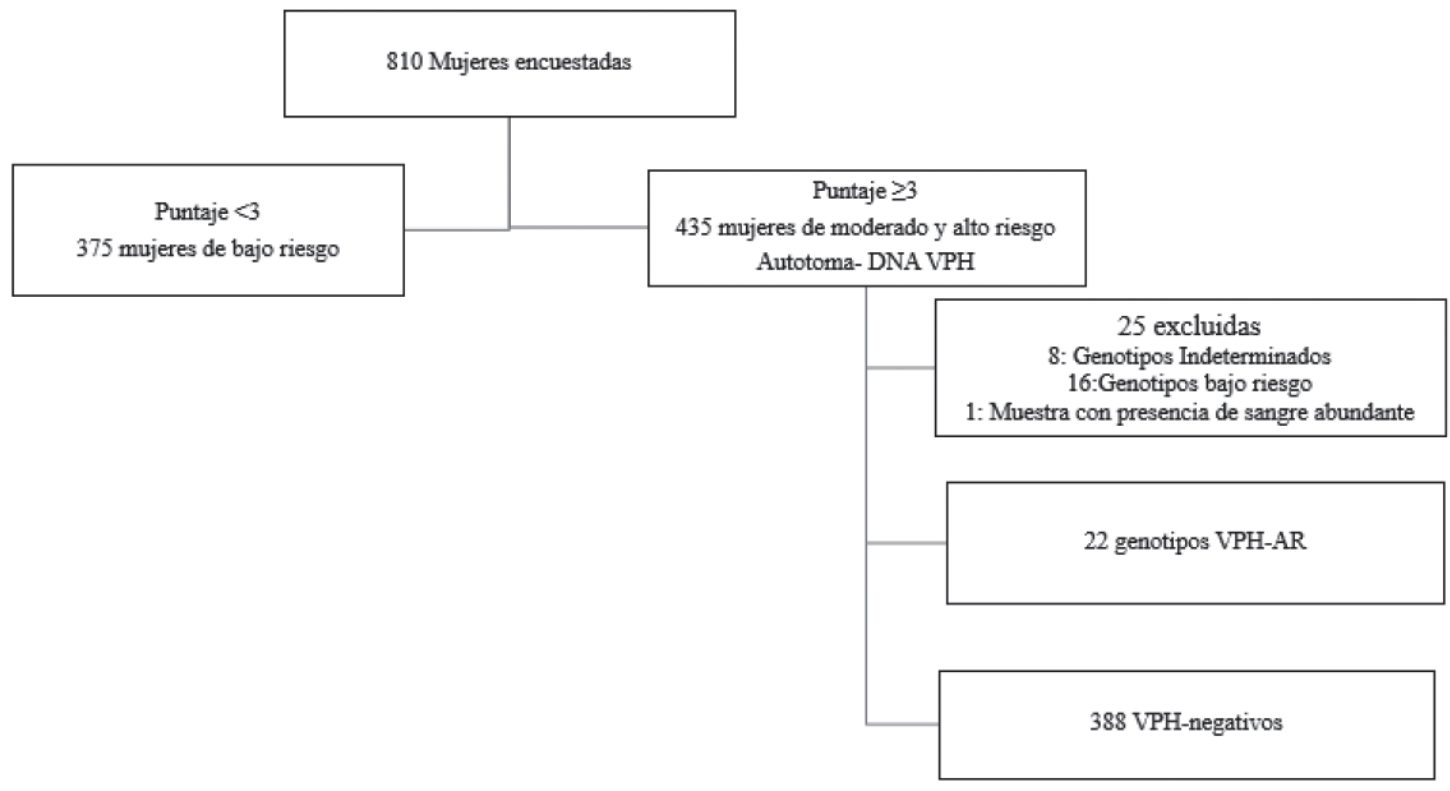

Figura 1. Captación de mujeres participantes en el estudio.

La prevalencia encontrada de infección por VPH-AR en las 435 mujeres incluidas en este estudio fue de 5,1\% (IC 95\% 3,2-7,6). Para evaluar los factores asociados a la infección por VPH se tomaron las mujeres infectadas por al menos un genotipo de VPH-AR y aquellas sin infección (VPH negativas), para una muestra de 410 mujeres.

En las características sociodemográficas, entre las mujeres no infectadas y las infectadas con VPH-AR no se presentaron diferencias estadísticamente significativas para la edad, el estado reproductivo, el estado civil, la ocupación, el nivel de escolaridad, el estrato de la vivienda o el número de personas que cohabitan (Tabla 1). Al evaluar la prevalencia de VPH-AR por decenios se observó que fue mayor en el grupo de 55 o más años, sin embargo, esto no fue estadísticamente significativo $(p=0,18)$ (Figura 2).

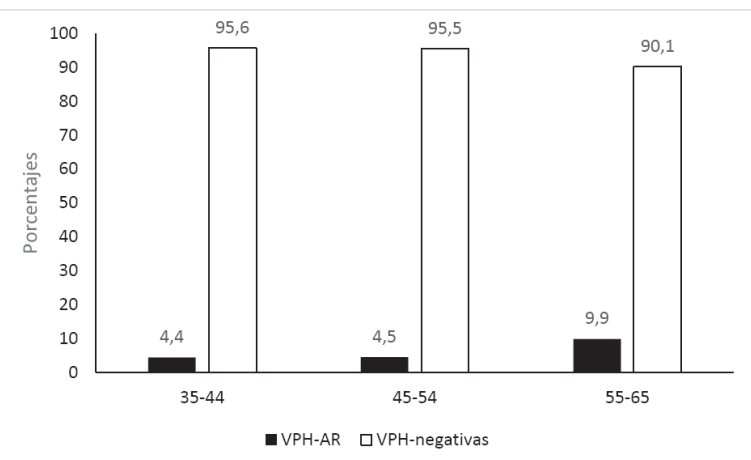

Figura 2. Prevalencia por decenios de infección por VPH$\mathrm{AR}^{\mathrm{a}}$ en mujeres de riesgo moderado/alto para $\mathrm{CCU}^{\mathrm{b}}$.
Respecto a factores ginecológicos no se encontraron diferencias significativas entre los grupos en la edad de inicio de las relaciones sexuales y número de compañeros sexuales. Aunque la frecuencia de enfermedades de trasmisión sexual durante su vida fue mayor en las mujeres con VPH-AR (36,3\% vs $25,7 \%$ ), esto tampoco fue estadísticamente significativo. Por otra parte, el acceso a la citología en los últimos tres años fue de $75,3 \%$ para el grupo en general (Tabla 1). Al considerar los métodos anticonceptivos, la proporción de mujeres, que han utilizado estas estrategias en algún momento de la vida fue de $88,4 \%$, entre estos se reportaron con frecuencia los anticonceptivos orales (ACO) $(46,2 \%)$ y los anticonceptivos inyectables (ACI) (27,6\%). Sin embargo, en la actualidad la ligadura de trompas fue el más utilizado $(60,4 \%)$ y aunque fue menos frecuente en el grupo con VPH-AR, no fue estadísticamente significativo. Por otra parte, se observó que el uso actual de ACI, de ACO y de dispositivo intrauterino (DIU) fue más frecuente en las mujeres infectadas, pero no fueron significativas estás diferencias. Los métodos menos utilizados en la actualidad fueron el condón $(1,7 \%)$ y el implante $(0,7 \%)$.

La presencia actual de síntomas vaginales fue más prevalente en las mujeres sin infección por VPH (Tabla 1). El síntoma más frecuente fue la rasquiña vaginal, con mayor prevalencia en las mujeres sin infección por VPH $(p=0,05)$; siguen en frecuencia el flujo vaginal $(24,4 \%)$, flujo con mal olor $(12,2 \%)$ y el sangrado vaginal $(6,1 \%)$. Otros síntomas como dolor pélvico e inflamación fueron reportados menos frecuentemente. 
Sedentarismo y Sobrepeso/Obesidad asociados a la prevalencia de infección por Virus de Papiloma Humano de alto riesgo en mujeres de la zona norte de Bucaramanga

Tabla 1. Descripción de las mujeres de riesgo moderado/alto para CCUb según el estado de infección por VPH-AR ${ }^{\mathrm{a}}$.

\begin{tabular}{|c|c|c|c|c|}
\hline Característica & $\begin{array}{c}\text { Total } \\
(\mathrm{n}=\mathbf{4 1 0})\end{array}$ & $\begin{array}{l}\text { VPH-AR positivos } \\
\quad(n=22)\end{array}$ & $\begin{array}{l}\text { VPH-AR negativos } \\
\quad(\mathbf{n}=\mathbf{3 8 8})\end{array}$ & $\mathbf{p}$ \\
\hline Edad mediana (RIQ) & $46(40-52)$ & $47(40-55)$ & $46(40-52)$ & 0,59 \\
\hline Estado civil n (\%) & $\mathrm{n}=406$ & $\mathrm{n}=22$ & $\mathrm{n}=384$ & \\
\hline Parejas estables & $277(68,2)$ & $18(81,8)$ & $259(67,4)$ & 0,15 \\
\hline Ocupación & $\mathrm{n}=406$ & $\mathrm{n}=22$ & $n=384$ & \\
\hline Primordialmente en la vivienda & $235(57,8)$ & $16(72,7)$ & $219(57,0)$ & 0,14 \\
\hline Años de estudios cursados & $\begin{array}{c}n=383 \\
6,2(3-9)\end{array}$ & $\begin{array}{c}n=20 \\
5,7(3-10)\end{array}$ & $\begin{array}{c}n=363 \\
6,2(4-9)\end{array}$ & 0,39 \\
\hline Estrato de vivienda & $\mathrm{n}=406$ & $\mathrm{n}=22$ & $\mathrm{n}=384$ & \\
\hline Estrato uno & $282(69,5)$ & $16(72,7)$ & $266(69,2)$ & \\
\hline Estrato dos & $119(29,3)$ & $6(27,2)$ & $113(29,5)$ & 0,94 \\
\hline Estrato tres & $2(0,5)$ & $0(0,0)$ & $2(0,6)$ & \\
\hline Sin clasificación & $3(0,7)$ & $0(0,0)$ & $3(0,8)$ & \\
\hline Seguridad social & $\mathrm{n}=406$ & $\mathrm{n}=22$ & $\mathrm{n}=384$ & \\
\hline Subsidiado & $352(86,7)$ & $19(86,3)$ & $333(86,7)$ & \\
\hline Contributivo & $46(11,3)$ & $3(13,6)$ & $43(11,2)$ & 0,90 \\
\hline No asegurado & $7(1,7)$ & $0(0,0)$ & $7(1,8)$ & \\
\hline Especial & $1(0,2)$ & $0(0,0)$ & $1(0,2)$ & \\
\hline Número de personas que habitan en casa mediana (RIQ) & $\begin{array}{c}n=405 \\
4,5(3-6)\end{array}$ & $\begin{array}{c}n=22 \\
5,0(3-6)\end{array}$ & $\begin{array}{c}n=383 \\
4,4(3-6)\end{array}$ & 0,83 \\
\hline Edad primera relación sexual $<16$ años & $247(60,2)$ & $14(63,7)$ & $233(60,0)$ & 0,73 \\
\hline Tres o más compañeros sexuales & $364(88,8)$ & $19(86,3)$ & $345(89)$ & 0,71 \\
\hline Edad del primer embarazo mediana(RIQ) & $\begin{array}{c}n=403 \\
18,2(16-20)\end{array}$ & $\begin{array}{c}n=21 \\
18,4(17-20)\end{array}$ & $\begin{array}{c}n=382 \\
18,2(16-20)\end{array}$ & 0,47 \\
\hline Número de embarazos totales & $\begin{array}{c}n=405 \\
4,4(3-5)\end{array}$ & $\begin{array}{c}n=21 \\
4,7(4-5)\end{array}$ & $\begin{array}{c}n=384 \\
4,4(3-5)\end{array}$ & 0,44 \\
\hline Número de hijos nacidos vivos & $\begin{array}{c}\mathrm{n}=405 \\
3,8(3-5)\end{array}$ & $\begin{array}{c}n=21 \\
4,4(3-5)\end{array}$ & $\begin{array}{c}\mathrm{n}=384 \\
3,8(3-5)\end{array}$ & 0,21 \\
\hline Números de abortos & $\begin{array}{c}\mathrm{n}=405 \\
0,5(0-1)\end{array}$ & $\begin{array}{c}\mathrm{n}=21 \\
0,2(0-0)\end{array}$ & $\begin{array}{c}\mathrm{n}=384 \\
0,5(0-1)\end{array}$ & 0,12 \\
\hline Relaciones sexuales orales $n(\%)$ & $90(21,9)$ & $2(9,0)$ & $88(22,6)$ & 0,13 \\
\hline Relaciones sexuales anales & $41(10,0)$ & $1(4,5)$ & $40(10,3)$ & 0,38 \\
\hline Antecedente de ETS & $108(26,3)$ & $8(36,3)$ & $100(25,7)$ & 0,48 \\
\hline Citología en los últimos 3 años & $\begin{array}{c}n=409 \\
308(75,3)\end{array}$ & $\begin{array}{c}\mathrm{n}=22 \\
18(81,9)\end{array}$ & $\begin{array}{c}n=387 \\
290(75)\end{array}$ & 0,75 \\
\hline Ligadura de trompas & $\begin{array}{c}n=405 \\
245(60,4)\end{array}$ & $\begin{array}{c}n=22 \\
10(45,4)\end{array}$ & $\begin{array}{c}n=383 \\
235(61,3)\end{array}$ & 0,13 \\
\hline Actual uso de ACI & $\begin{array}{l}n=403 \\
13(3,2)\end{array}$ & $\begin{array}{l}\mathrm{n}=22 \\
2(9,0)\end{array}$ & $\begin{array}{l}n=381 \\
11(2,8)\end{array}$ & 0,10 \\
\hline Síntomas vaginales & $213(51,9)$ & $5(22,7)$ & $208(53,6)$ & 0,017 \\
\hline Rasquiña vaginal & $\begin{array}{c}\mathrm{n}=409 \\
111(27,1)\end{array}$ & $\begin{array}{c}n=22 \\
2(9,09)\end{array}$ & $\begin{array}{c}\mathrm{n}=387 \\
109(28,1)\end{array}$ & 0,050 \\
\hline $\begin{array}{l}\text { Tabaquismo } \\
\text { Nunca } \\
\text { Si fuma } \\
\text { Fumaba, pero lo dejé }\end{array}$ & $\begin{array}{l}257(62,6) \\
39(9,5) \\
114(27,8)\end{array}$ & $\begin{array}{l}12(54,6) \\
2(9,0) \\
8(36,3)\end{array}$ & $\begin{array}{c}245(63,1) \\
37(9,5) \\
106(27,3)\end{array}$ & 0,65 \\
\hline $\begin{array}{l}\text { IMC } \\
\text { Normal } \\
\text { Sobrepeso/obesas }\end{array}$ & $\begin{array}{l}n=379 \\
106(28) \\
273(72)\end{array}$ & $\begin{array}{c}\mathrm{n}=22 \\
2(9,1) \\
20(90,9)\end{array}$ & $\begin{array}{c}\mathrm{n}=357 \\
104(29,1) \\
253(70,9)\end{array}$ & 0,042 \\
\hline $\begin{array}{l}\text { Sedentarismo } \\
\text { Actividad física total }<150^{\prime} \text { ' por semana } \\
\text { Actividad física total } \geq 150^{\prime} \text { por semana }\end{array}$ & $\begin{array}{c}\mathrm{n}=406 \\
114(28,0) \\
292(72,0)\end{array}$ & $\begin{array}{c}n=22 \\
11(50,0) \\
11(50,0)\end{array}$ & $\begin{array}{c}n=384 \\
103(27,0) \\
281(73,0)\end{array}$ & 0,01 \\
\hline $\begin{array}{l}\text { No. de porciones de frutas y verduras consumidas/día } \\
\text { mediana(RIQ) }\end{array}$ & $\begin{array}{c}\mathrm{n}=405 \\
1,65(0,5-3)\end{array}$ & $\begin{array}{c}\mathrm{n}=22 \\
1,32(0,28-1,28)\end{array}$ & $\begin{array}{c}\mathrm{n}=383 \\
1,6(0-5,3)\end{array}$ & 0,19 \\
\hline
\end{tabular}

${ }^{a}$ VPH-AR: Virus de Papiloma Humano de alto Riesgo

${ }^{\mathrm{b}}$ CCU: Cáncer de Cuello Uterino 
En cuanto a los hábitos, la mayoría de las mujeres reportaron nunca haber fumado (Tabla 1). Aunque la cantidad de cigarrillos fumados por día en las exfumadoras fue mayor en mujeres infectadas (mediana 21 cigarrillos día vs 6,9) y fumaron durante más años (12,7 años vs 9,2), estas diferencias no fueron significativas. Solo el $9,5 \%$ de las mujeres fuman actualmente con un consumo de 7,2 cigarrillos por día (RIQ 2-7), sin diferencias entre los dos grupos. Además, el tabaquismo dentro de la vivienda por parte de familiares fue reportado por el $40,9 \%$ de las mujeres con VPH-AR y por el $30,7 \%$ en las negativas. Al considerar el consumo de alcohol, el $68,8 \%$ de las mujeres manifestaron que nunca lo consumen y tomando como referencia la recomendación de la OMS, que define como consumo de riesgo a la ingesta de más de 40 gramos diarios de alcohol, se observó que ninguna tuvo este factor.

Hábitos saludables como el consumo de frutas y verduras también fueron evaluados a partir de la frecuencia y cantidades de consumo total de las mismas en la dieta. Teniendo en cuenta la recomendación de la OMS, que para una dieta saludable se precisa consumir cinco porciones al día, se observó un consumo deficiente en ambos grupos, con una mediana menor en las mujeres positivas para VPH-AR, aunque esto no fue significativo (Tabla 1).
Considerando que la actividad física moderada adecuada es definida por la OMS como la realización de al menos 150 minutos semanales de este tipo de actividades. Se encontró que el $50 \%$ de las mujeres con VPH-AR no realizan actividades físicas moderadas, en contraste con el $29,5 \%$ de las sin infección. Equivalente a ésta, la práctica de actividades vigorosa adecuada es considerada según la OMS como la realización de al menos 75 minutos semanales; se observó que ninguna de las infectadas por VPH-AR cumplía esta recomendación, similar al patrón observado en las mujeres negativas $(93,4 \%)$. Además, con estas variables se estimó la frecuencia de sedentarismo, observándose que la prevalencia fue significativamente mayor en las mujeres infectadas con VPH-AR; esto mismo se evidenció para la prevalencia de sobrepeso/obesidad (Tabla 1).

Posteriormente, con el modelo múltiple se determinó que el sedentarismo $(\mathrm{p}=0,021)$ y el sobrepreso/ obesidad $(\mathrm{p}=0,049)$ se asociaron independiente a mayor prevalencia de infección por VPH-AR. Adicionalmente, aunque el uso de anticonceptivos inyectados $(\mathrm{p}=0,062)$ presentó una tendencia de asociación con mayor prevalencia de infección por VPH, y el número de porciones de fruta/verdura $(\mathrm{p}=0,151)$ y la rasquiña vaginal $(\mathrm{p}=0,056)$ tuvieron una tedencia de asociación con menor prevalencia de infección, esto no fue estadísticamente significativo (Tabla 2).

Tabla 2. Factores asociados a la prevalencia de infección por VPH-AR ${ }^{\mathrm{a}}$ en mujeres con riesgo moderado/alto para CCU . $^{\mathrm{b}}$

\begin{tabular}{|c|c|c|c|c|c|}
\hline Característica & n & $\mathbf{R P}$ & IC $95 \%$ & $\mathbf{R P a}$ & IC 95\% \\
\hline $\begin{array}{l}\text { Estado civil } \\
\text { Pareja estable } \\
\text { Pareja no definida }\end{array}$ & 406 & $\begin{array}{c}1 \\
0,47\end{array}$ & $(0,16-1,38)$ & & \\
\hline $\begin{array}{l}\text { Ocupación } \\
\text { Ocupación en la vivienda } \\
\text { Otras }\end{array}$ & 406 & $\begin{array}{c}1 \\
0,51\end{array}$ & $(0,20-1,28)$ & & \\
\hline $\begin{array}{l}\text { Edad } \\
35-54 \text { años } \\
55-65 \text { años }\end{array}$ & 410 & $\begin{array}{c}1 \\
2,23\end{array}$ & $(0,94-5,26)$ & & \\
\hline Síntomas vaginales & 410 & 0,26 & $(0,10-0,71)$ & & \\
\hline Rasquiña vaginal & 409 & 0,26 & $(0,06-1,12)$ & 0,25 & $(0,06-1,04)$ \\
\hline Números de abortos & 405 & 0,57 & $(0,26-1,21)$ & & \\
\hline Ligadura de trompas & 405 & 0,54 & $(0,24-1,22)$ & & \\
\hline Actual uso de ACI & 403 & 3 & $(0,78-11,50)$ & 2,81 & $(0,95-8,34)$ \\
\hline Relaciones sexuales orales & 410 & 0,35 & $(0,08-1,49)$ & & \\
\hline Sedentarismo & 406 & 2,56 & $(1,14-5,74)$ & 2,51 & $(1,15-5,50)$ \\
\hline Sobrepeso/obesas & 379 & 3,88 & $(0,92-16,3)$ & 4,20 & $(1,00-17,51)$ \\
\hline Porciones diarias de fruta/verdura & 405 & 0,78 & $(0,53-1,13)$ & 0,77 & $(0,54-1,11)$ \\
\hline
\end{tabular}

${ }^{a}$ VPH-AR: Virus de Papiloma Humano de alto Riesgo

${ }^{\mathrm{b}} \mathrm{CCU}$ : Cáncer de Cuello Uterino 


\section{Discusion}

Se encuestaron en total 810 mujeres, quienes se clasificaron como de bajo riesgo $(46,3 \%)$ y riesgo moderado/alto $(53,7 \%)$ según la escala basada en el Siteman Cancer Center para CCU. Aunque la población en la que se realizó la detección molecular del VPH fue la compuesta por las mujeres con riesgo moderado/ alto para $\mathrm{CCU}$, se encontró una prevalencia baja de infección por VPH-AR $(5,1 \%$ IC95\% 3,2-7,6). Lo anterior contrasta con la prevalencia de $9 \%$ de VPH-AR reportada en Colombia en una cohorte de mujeres de 18 a 45 años de edad con citología normal; sin embargo, esta frecuencia es similar a la encontrada específicamente en las mujeres de 35 o más años de esta cohorte (infección VPH-AR única 4,3\% y múltiple $1,9 \%)^{18}$.

Las características sociodemográficas del grupo de mujeres infectadas con VPH-AR fueron similares a las del grupo en las que no se detectó ADN viral. De igual forma, no se encontraron diferencias entre ambos grupos de mujeres en otras variables ginecológicas como el número de embarazos, de nacidos vivos, entre otras. En cuanto a la prevalencia de vida del uso de métodos anticonceptivos, los ACI, ACO y DIU fueron las estrategias de prevención del embarazo más utilizados, y para el momento de la entrevista la ligadura de trompas fue el más frecuente. El uso del implante y del preservativo fueron métodos poco utilizados en la población objeto. Este bajo uso de preservativos remarca las deficiencias en la utilización de los métodos seguros de barreras para evitar la propagación de las infecciones de transmisión sexual.

Respecto a factores comportamentales, hábitos y dieta se observó poca frecuencia de consumo actual de alcohol y de cigarrillo, sin embargo un porcentaje importante de las mujeres infectadas reportaron ser exfumadoras y fumadoras pasivas. Adicionalmente, hábitos saludables como son el consumo de frutas y verduras fueron deficientes en relación con las recomendaciones internacionacionales de dieta saludable, ya que las porciones de frutas y verduras diarias fueron inferiores a las cinco porciones establecidas. Además, se encontró una prevalencia de sobrepeso/obesidad de $72 \%$ y una prevalencia de sedentarimo de $28 \%$.

Múltiples factores asociados a infección por VPH-AR se han descrito en la literatura, algunos controversiales porque varían de acuerdo con las regiones geográficas y a las características de las poblaciones. Por ejemplo, algunos estudios muestran que las infecciones cervicales se producen poco después de la primera relación sexual y el riesgo de tener VPH aumenta con cada nueva pareja sexual durante toda la vida. Al respecto, un estudio de corte transversal observó que mujeres estadounidenses de origen mexicano entre 17 a 47 años que iniciaron su vida sexual antes de los 16 años tuvieron mayor riesgo de adquirir infección por VPH-AR (ORa 1,58 IC95\% 1,09-2,30) en comparación con aquellas que iniciaron la actividad sexual después de los 20 años ${ }^{19}$. En contra posición pero similar a lo encontrado en el presente estudio, en una cohorte de 1.373 colombianas entre 13 y 85 años, mujeres menores de 16 años que habían iniciado la vida sexual no tuvieron mayor riesgo de infección por VPH-AR (ORa 0,7 IC95\% 0,52-1,06) comparadas con las mujeres con inicio de actividad sexual después de los 19 años ${ }^{20}$.

Además de la edad temprana de inicio de la actividad sexual, se ha estudiado la asociación entre el número de compañeros sexuales y la infección por VPHAR. Al respecto, en la presente investigación no se encontró esta asociación, similar a lo reportado en un estudio realizado en una zona rural de Colombia. En éste, el número de compañeros sexuales ocasionales mayor a dos durante la vida no estuvo asociado con la seropositividad para los genotipos VPH-AR 16, 18, 31 o 58 (ORa 1,08 IC95\% 0,72-1,63) cuando se comparó con aquellas mujeres que reportaron una sola pareja sexual oscasional durante su vida ${ }^{21}$.

Otro factor evaluado son las infecciones de transmisión sexual, en nuestro país recientemente se determinó que en mujeres con citología normal la prevalencia de infección por Chlamydia trachomatis fue mayor en aquellas con infección por varios genotipos de VPH (OR; 2,8 IC95\% 1,2-6,0), comparadas con aquellas que solo presentaban infección por un genotipo de $\mathrm{VPH}^{22}$. En el presente estudio no se evaluó la infección concomitante, pero, aunque la frecuencia del antecedente de ETS fue mayor en las mujeres con VPH-AR, esto no fue estadísticamente significativo. Esto podría deberse al desconocimiento que tienen las mujeres sobre las infecciones de transmisión sexual lo que impide reconocer la presencia de las mismas.

El uso de anticonceptivos hormonales también es un factor controversial de infección por VPH. Por ejemplo, en Brasil un estudio no encontró asociación con el consumo de ACO (OR 1,49; IC95\% 0,48-4,57) Por el contrario, en la cohorte de mujeres colombianas este factor se asoció con infección por VPH (OR 1,38; IC95\% 1,07-1,77) ${ }^{20}$. En el presente estudio se observó que el uso actual de ACI fue más prevalente en las mujeres con infección por VPH-AR, sin embargo, 
presentó una asociación marginal probablemente por falta de poder (RPa 2,81; IC95\% 0,95-8,34). Además, esta discrepancia en los resultados puede deberse a diferencias en las poblaciones como la edad, el comportamiento sexual, tabúes al momento de hablar sobre sexualidad, así como los métodos y técnicas empleadas en cada uno de los estudios ${ }^{20}$.

En cuanto a los hábitos, el consumo de cigarrillo se ha relacionado con aumento en la prevalencia de infección por VPH-AR, esta asociación parece ser dependiente de la dosis y desaparece luego de suspender el consumo ${ }^{23}$. En nuestro estudio el tabaquismo actual fue similar entre los grupos y el tabaquismo previo, aunque fue reportado en mayor frecuencia en las mujeres infectadas por VPH-AR no fue significativo.

La dieta también ha sido considerada dentro de los factores asociados a la infección por VPH. En este estudio, aunque la mayoría de mujeres no cumplieron con la recomendación de consumo de cinco porciones diarias, se observó una tendencia de disminución de la prevalencia de infección por VPH-AR a medida que se incrementan las porciones de fruta y verduras consumidas durante el día (RPa 0,77; IC95\% 0,54-1,11). Esto podría relacionarse al consumo de vitaminas, ya que un estudio de cohorte, que evaluó el papel de la vitamina A y los carotenoides en la persistencia de VPH, encontró que el alto consumo de verduras se asoció con una disminución de la persistencia de VPH (OR 0,46; IC95\% 0,21- 0,97 $)^{24}$.

En el presente estudio también se evaluó el IMC, encontrando una asociación entre la infección con VPH-AR y el sobrepeso/obesidad (PRa 4,20; IC95\% $1,00-15,51)$. Este factor se había reportado previamente asociado a desarrolló de CCU, ya que las mujeres con IMC $\geq 25 \mathrm{~kg} / \mathrm{m}^{2}$ tuvieron mayor posibilidad de desarrollar este cáncer (OR1,70; IC95\% 1,10-2,63) comparadas con el grupo mujeres con IMC normal $\left(18,5-23 \mathrm{~kg} / \mathrm{m}^{2}\right)^{25}$.

Por último, este estudio encontró que las mujeres sedentarias tuvieron mayor prevalencia de infección por VPH-AR (RPa 2,51; IC95\% 1,15-5,50) en relación a las que notificaron actividad física total de 150 minutos o más de actividad moderada o su equivalente en actividad vigorosa por semana, según la definición de la OMS. Al respecto, un estudio que midió la actividad física de tiempo libre en MET/horas/semana encontró que la actividad física alta ( $\geq 75 \mathrm{MET} /$ horas/semana) fue factor protector para la aparición de CCU (ORa 0,61; IC95\% 0,38-0,89) en comparación con la actividad física baja (<38,5 MET/horas/semana), aunque no hubo asociación con actividad física media (38,5-71,9 MET/ horas/semana) (ORa 0,95; IC95\% 0,61-1,48) ${ }^{25}$.

Entre las limitaciones encontradas en este estudio, la principal es el tamaño de la muestra, ya que por la baja prevalencia encontrada sólo se detectaron 22 infecciones, lo que dificultó establecer asociaciones entre la infección por VPH-AR y los diferentes factores de riesgo evaluados, como la edad y el uso de anticonceptivos. Otra limitación fue la falta de detección de DNA de VPH en las mujeres con puntaje menor de tres en la escala de riesgo para CCU basada en Siteman Cancer Center. Esto posiblemente ocasionó la homogenización de las variables que hacen parte de la escala en las mujeres diagnosticadas, limitando la evaluación de estos potenciales factores. Sin embargo, cabe anotar que esta tamización estuvo condicionada a la optimización de los recursos económicos del proyecto, lo cual se apoya en el hecho de la baja prevalencia de infección por VPH-AR encontrada, pese a que se evaluaron sólo las mujeres con riesgo moderado/alto de CCU.

En conclusión, se logró establecer asociaciones no antes descritas en nuestro país, ya que se observó que el sobrepeso/obesidad y el sedentarismo fueron factores independientemente asociados a una mayor prevalencia de infección por VPH-AR. Esta investigación aporta nueva información para la creación de estrategias complementarias que incluyan estos dos factores modificables que no están relacionados con el comportamiento sexual y que dependen exclusivamente de la mujer. Estas nuevas estrategias podrían impactar en la prevención o eliminación temprana de la infección por VPH-AR en las mujeres de nuestra región.

Adicionalmente se determinaron otros factores que podrían estar asociados con menor prevalencia de infección por VPH-AR como la dieta con alto consumo de frutas y verduras, así como factores que podrían estar relacionados con mayor prevalencia de infección por VPH-AR como el uso de ACI. Sin embargo, se requieren estudios con mayor tamaño de muestra para comprobar estas potenciales asociaciones en nuestra población.

\section{Agradecimientos}

A las participantes que permitieron el desarrollo de esta investigación, MinCiencias por su financiación a través del proyecto código: 110272553474, Universidad Industrial de Santander, ISABU, líderes comunitarios y Centro de Salud de la zona norte de Bucaramanga. 


\section{Consideraciones éticas}

El presente estudio se encuentra anidado a un estudio marco que contó con la aprobación por parte del comité de ética del Hospital Local del Norte, para el cual se tomó consentimiento informado.

\section{Conflicto de interés}

No existe conflicto de interés.

\section{Referencias}

1. De Villiers EM, Fauquet C, Broker TR, Bernard HU, Zur Hausen H. Classification of papillomaviruses. Virology. 2004; 324(1): 17-27. doi: 10.1016/j. virol.2004.03.033.

2. Longworth MS, Laimins LA. Pathogenesis of Human Papillomaviruses in differentiating epithelia. Microbiol Mol Biol Rev. 2004; 68(2): 362-372. doi: 10.1128/MMBR.68.2.362-372.2004.

3. Ferlay J, Soerjomataram I, Ervik M, Dikshit R, Eser S, Mathers C, et al. GLOBOCAN 2012 v1.0, Cancer Incidence and Mortality Worldwide: IARC CancerBase. International Agency for Research on Cancer. 2013; (11).

4. Arbyn M, Castellsagué X, de Sanjosé S, Bruni L, Saraiya M, Bray F, et al. Worldwide burden of cervical cancer in 2008. Ann Oncol. 2011; 22(12): 2675-2686. doi: 10.1093/annonc/mdr015.

5. de Martel C, Plummer M, Vignat J, Franceschi S. Worldwide burden of cancer attributable to HPV by site, country and HPV type. Int J Cancer. 2017; 141(4): 664-670. doi: 10.1002/ijc.30716.

6. Ribeiro AA, Costa MC, Alves RRF, Villa LL, Saddi VA, Carneiro MADS, et al. HPV infection and cervical neoplasia: Associated risk factors. Infect Agent Cancer. 2015;10(1). doi: 10.1186/s13027015-0011-3.

7. Hernandez BY, Wilkens LR, Zhu X, Thompson P, McDuffie K, Shvetsov YB, et al. Transmission of human papillomavirus in heterosexual couples. Emerg Infect Dis. 2008; 14(6): 888-894. doi: 10.3201/eid1406.070616.2.

8. Vielot N, Hudgens MG, Mugo N, Chitwa M, Kimani J, Smith J. The role of chlamydia trachomatis in high-risk human papillomavirus persistence among female sex workers in Nairobi, Kenya. Sex Transm Dis. 2015; 42(6): 305-311. doi: 10.1097/ OLQ.0000000000000287.

9. Hwang LY, Ma Y, Benningfield SM, Clayton L, Hanson EN, Jay J, et al. Factors that influence the rate of epithelial maturation in the cervix in healthy young women. J. Adolesc Heal. 2009; 44(2): 103110. doi: 10.1016/j.jadohealth.2008.10.006.

10. Stensen S, Kjaer SK, Jensen SM, Frederiksen K, Junge $\mathrm{J}$, Iftner $\mathrm{T}$, et al. Factors associated with type-specific persistence of high-risk human papillomavirus infection: a population-based study. Int J Cancer. 2016; 138(2): 361-368. doi: 10.1002/ ijc.29719.

11. Obiri YD, Adu SY, Djigma F, Hayfron B, Abdul L, Simpore J, Mayaud, P, et al. Self-collected vaginal sampling for the detection of genital human papillomavirus (HPV) using care HPV among Ghanaian women. BMC women's health. 2017; 17(1), 86. doi: 10.1186/s12905-017-0448-1.

12. Gupta SP, Bik EM, Cardenas JP, Nuñez H, Kraal $\mathrm{L}$, Goddard AD, et al. Self-sampling for human papillomavirus testing: increased cervical cancer screening participation and incorporation in international screening programs. Front Public Health. 2018; 6: 77. doi: 10.3389/fpubh.2018.00077.

13. Ketelaars PJ, Bosgraaf RP, Siebers AG, Massuger LG, Van der Linden JC, Wauters CA, et al. High-risk human papillomavirus detection in self-sampling compared to physician-taken smear in a responder population of the Dutch cervical screening: results of the VERA study. Prev Med. 2017; 101: 96-101. doi: 10.1016/j.ypmed.2017.05.021.

14. Jentschke M, Chen K, Arbyn M, Hertel B, Noskowicz M, Soergel P, et al. Direct comparison of two vaginal self-sampling devices for the detection of human papillomavirus infections. J Clin Virol. 2016; 82: 46-50. doi: 10.1016/j.jcv.2016.06.016.

15. Herraez-Hernandez E, Alvarez-Perez M, NavarroBustos G, Esquivias J, Alonso S, Aneiros-Fernandez J, et al. HPV Direct Flow CHIP: A new human papillomavirus genotyping method based on direct PCR from crude-cell extracts. J Virol Methods. 2013; 193(1) :9-17. doi: 10.1016/j.jviromet.2013.04.018.

16. Eklund CF, Forslund O, Wallin KL, Dillner J. Global improvement in genotyping of human papillomavirus DNA: the 2011 HPV LabNet International Proficiency Study. J Clin Microbiol. 2014; 52(2); 449-459. doi: 10.1128/JCM.02453-13.

17.2008 Physical activity guidelines advisory committee. Physical activity guidelines advisory committee report. Washington DC: US. 2008; 67(2): 683.

18. Molano M, Posso H, Weiderpass E, Van den Brule AJC, Ronderos M, Franceschi S, et al. Prevalence and determinants of HPV infection among Colombian women with normal cytology. Br J Cancer. 2002; 87(3): 324-333. doi: 10.1038/sj.bjc.6600442.

19. Giuliano AR, Papenfuss M, Schneider A, Nour M, 
Hatch K. Risk factors for high-risk type human papillomavirus infection among Mexican-American women. Cancer Epidemiol Biomarkers Prev. 1999; 8(7): 615-620.

20. Molano M, Van den Brule A, Plummer M, Weiderpass E, Posso H, Arslan A, et al. Determinants of clearance of human papillomavirus infections in Colombian women with normal cytology: A population-based, 5-year follow-up study. Am J Epidemiol. 2003; 158(5): 486-494.

21. Bedoya AM, Gaviria AM, Baena A, Borrero M, Duarte DF, Combita AL, et al. Age-specific seroprevalence of human papillomavirus 16, 18, 31 , and 58 in women of a rural town of Colombia. Int J Gynecol Cancer. 2012; 22(2): 303-310. doi: 10.1097/IGC.0b013e31823c2469.

22. Quinónez-Calvache EM, Ríos-Chaparro DI, Ramírez JD, Soto-De León SC, Camargo M, Río-Ospina L Del, et al. Chlamydia trachomatis Frequency in a Cohort of HPV-infected Colombian women. PLoS One. 2016; 11(1). doi: 10.1371/ journal.pone.0147504.

23. Appleby P, Beral V, Berrington de González A, Colin D, Franceschi S, Goodill A, et al. Carcinoma of the cervix and tobacco smoking: Collaborative reanalysis of individual data on 13,541 women with carcinoma of the cervix and 23,017 women without carcinoma of the cervix from 23 epidemiological studies. Int J Cancer. 2006; 118(6): 1481-1495. doi: 10.1002/ijc. 21493.

24. Giuliano AR, Harris R, Sedjo RL, Baldwin S, Roe $\mathrm{D}$, Papenfuss MR, et al. Incidence, prevalence, and clearance of type-specific human papillomavirus infections: The Young Women's Health Study. J Infect Dis. 2002; 186(4): 462-469. doi: $10.1086 / 341782$.

25. Lee JK, So KA, Piyathilake CJ, Kim MK. Mild Obesity, Physical Activity, Calorie intake, and the risks of cervical intraepithelial neoplasia and cervical cancer. PLoS One. 2013; 8(6). doi: 10.1371/ journal.pone.0066555. 